\title{
La protection des captages et les intérêts agricoles (Restrictions à l'usage de l'eau)
}

\section{The water catchment protection and the agriculture stakes}

\author{
par Bernard Peignot \\ Avocat aux Conseils \\ Secrétaire général de l'AFDR \\ Professeur à l'Institut des Hautes Etudes de Droit Rural et d'Economie Agricole \\ (IHEDREA)
}

Facing the pollution emitted by agricultural activities, the law attempts to intervening at the beginning in order to protect the drinking water resources by erecting "constraining judicial barriers", which are constituted by protective perimeters around the water catchments.

Often considered as insufficient, these measures have to give way to more flexible and varied proceedings, transiting via a direct mediation of the landowner on the agricultural activities themselves.

\section{I — INTRODUCTION}

La protection de l'eau, spécialement de celle destinée à l'alimentation humaine, est devenue un enjeu national et international, face aux pollutions, en particulier par les nitrates, d'origine agricole.

Se référant au rapport de Monsieur Philippe MANGIN $[1,2]$, le Conseil Economique et Social a déjà eu dans le passé (en 1991), l'occasion de dénoncer cette pollution lente et pernicieuse, constitutive d'un véritable danger à long terme, pour la ressource en eau, considérée comme patrimoine commun de la Nation [3].

Face à cette menace, une Directive Européenne du 12 décembre 1991 ( $\mathrm{n}^{\circ}$ 91-676) révisée en novembre 1997, a organisé, par l'adoption de programmes d'action, la réduction de cette pollution. Chargés de sa transposition au plan interne, le législateur et les Pouvoirs Publics ont donc amélioré par des interventions, les mesures de protection de la ressource.

Mais qui dit bien commun, n'implique pas que n'importe qui peut disposer en toute liberté de cette ressource précieuse qu'est devenue l'eau [4]. Pourtant parce qu'elle n'appartient à personne, l'eau destinée à la consommation humaine, valeur environnementale par excellence, ne peut être protégée qu'au regard de celui qui l'exploite.

En ce sens, le droit peut agir de deux manières : soit, pour protéger la ressource contre les usages du sol, en dressant des "barrières juridiques contraignantes" ; ainsi en va-t-il des périmètres de protection autour des captages qui, de proche en proche, limitent ou interdisent les activités polluantes notamment d'origine agrieole (voir § II).
Mais considérées à la longue et à juste titre comme insuffisantes, ces mesures doivent laisser la place à des procédés plus souples et variés, passant par une médiation directe du propriétaire du sol sur les activités elles-mêmes, dès lors qu'elles sont susceptibles d'entrer en conflit avec l'usage particulier, l'alimentation humaine, assigné à la ressource en eau (voir § III).

\section{II $\square$ LA MISE EN PLACE DES PÉRIMÈTRES DE PROTECTION DES CAPTAGES [5]}

L'une des responsabilités importantes des collectivités est de distribuer une eau de bonne qualité chimique et bactériologique, conforme aux normes de "potabilité" compatibles avec la Santé Publique [6].

Aussi convient-il d'assurer la protection des captages contre les pollutions de surface chroniques ou accidentelles, en déterminant autour d'eux des périmètres de protection dans lesquels certaines activités peuvent être interdites ou réglementées : c'est la protection de l'intérêt général qui guide le législateur et le pouvoir réglementaire dans leur œuvre normative, qu'il s'agisse de l'eau dite d'alimentation ou de l'eau minérale.

\subsection{L'eau dite "d'alimentation"}

\subsubsection{Le cadre juridique}

Introduite pour la première fois par l'article 10 de la loi du 15 février 1902, en ce qui concerne les seuls captages de sources, l'institution des périmètres de protection n'a été 
rendue obligatoire que par un décret du 30 octobre 1935. En réalité ce texte ne sera que très peu appliqué et il faudra attendre la loi du 16 décembre 1964 (article 7) pour voir, à nouveau, dans un texte législatif spécifique, consacrer la nécessité de mettre en place des périmètres de protection.

Codifié à l'article L. 20 du code de la Santé Publique, le texte précise "qu'en vue d'assurer la protection de la qualité des eaux, l'acte portant déclaration d'utilité publique des travaux de prélèvement d'eau destinée à l'alimentation des collectivités humaines détermine autour du point de prélèvement un périmètre de protection immédiat, dont les terrains sont à acquérir en pleine propriété, un périmètre de protection rapproché à l'intérieur duquel peuvent être interdites ou réglementées toutes activités ou dépôts de nature à nuire à la qualité des eaux et le cas échéant un périmètre de protection éloigné à l'intérieur duquel peuvent être réglementées les activités et installations ci-dessus".

Des actes déclaratifs d'utilité publique peuvent encore dans les mêmes conditions, déterminer les périmètres de protection autour des points de prélèvements déjà existants, ainsi qu'autour des ouvrages d'adduction à écoulement libre et des réservoirs enterrés.

Mais la possibilité de définir de tels périmètres de protection autour des points de prélèvement d'eau mis en service avant la publication de la loi de 1964 étant restée facultative, le législateur (article 13 de la loi du 3 janvier 1992) complétant l'article L 20 du code de la Santé Publique a rendu obligatoire l'institution des périmètres de protection, et précisé qu'ils devaient être déterminés par déclaration d'utilité publique avant le 4 janvier 1997, (à cette date à peine un quart des captages, sur les 40000 que compte la France, bénéficie d'une protection effective) [7].

Un décret du 3 janvier 1989 (modifié) relatif aux eaux destinées à la consommation humaine donne à l'article 16 des précisions sur l'établissement de trois périmètres de protection et sur les interdictions possibles dans les deux premiers périmètres et les réglementations dans le périmètre de protection éloignée.

Une circulaire interministérielle du 24 juillet 1990 "relative à la mise en place des périmètres de protection des points de prélèvement d'eau destinée à la consommation humaine (article L20 du Code de la Santé Publique)" rappelle que l'instauration de périmètres de protection autour des points de prélèvement, dans les conditions définies par l'article L 20 du code de la Santé Publique, constitue un moyen efficace pour faire obstacle à des pollutions par des substances susceptibles d'altérer de façon notable la qualité des eaux prélevées.

A cette circulaire est jointe une instruction technique qui, pour l'essentiel, rappelle les principes fondamentaux à retenir pour l'établissement des périmètres de protection, et précise la procédure applicable en la matière, la nature des prescriptions pouvant être mises en oeuvre et les modalités de leur intégration dans les documents d'urbanisme.

Enfin, récemment, une circulaire du 2 janvier 1997 est venue rappeler aux communes leurs obligations en la matière, et a attiré leur attention sur les risques du non-respect des dispositions relatives aux périmètres de protection.

\subsubsection{Les périmètres de protection}

- Les périmètres de protection immédiate : ils ont pour fonction d'empêcher la détérioration des ouvrages de prélèvement et d'éviter que des déversements ou des infiltrations de substances polluantes se produisent à l'intérieur ou à proximité immédiate du captage. Leurs limites sont établies afin de prévenir toute introduction directe de substances polluantes dans les ouvrages.

Les terrains inclus dans ce périmètre sont acquis en pleine propriété, clôturés sauf dérogations prévues dans l'acte déclaratif d'utilité publique et régulièrement entretenus. Toutes activités, installations et dépôts y sont interdits, en dehors de ceux explicitement autorisés dans l'acte déclaratif d'utilité publique.

- les périmètres de protection rapprochée : ils doivent protéger efficacement le captage vis-à-vis de la migration souterraine des substances polluantes. Leur étendue est déterminée en prenant notamment en compte : les caractéristiques physiques de l'aquifère et de l'écoulement souterrain, le débit maximal de pompage, la vulnérabilité, l'origine et la nature des pollutions contre lesquelles il est nécessaire de protéger les eaux souterraines.

A l'intérieur des périmètres de protection rapprochée, peuvent être instaurées diverses servitudes et mesures de police sous forme d'interdiction et de réglementation.

- Les périmètres de protection éloignée : ils prolongent éventuellement les précédents pour renforcer la protection contre les pollutions permanentes ou diffuses. Ils sont créés si l'on estime que l'application de la réglementation générale, même renforcée, n'est pas suffisante, en particulier s'il existe un risque potentiel de pollution que la nature des terrains traversés ne permet pas de réduire en toute sécurité malgré l'éloignement du point de prélèvement.

A l'intérieur des périmètres de protection éloignée, peuvent être réglementés les activités, dépôts, ou installations qui présentent un danger de pollution pour les eaux prélevées, par la nature et la quantité des produits polluants mis en jeu, ou par l'étendue des surfaces qu'ils affectent.

\subsection{Les sources d'eau minérale}

L'eau minérale est définie comme une eau possédant un ensemble de caractéristiques qui sont de nature à lui apporter des propriétés favorables à la santé.

Provenant d'une nappe ou d'un gisement souterrain, elle se distingue des autres eaux destinées à la consommation humaine notamment par sa nature, caractérisée par sa teneur en sels minéraux, en oligo-éléments et autres constituants. Elle ne peut faire l'objet d'aucun traitement ou adjonction autres que ceux autorisés par arrêté ministériel (article 12 du décret du 6 juin 1989).

La protection des eaux minérales est assurée par la déclaration d'intérêt public, prononcée par décret en Conseil d'Etat, et par la procédure de périmètre de protection, prévue par l'article L 736 du Code de la Santé Publique.

Le périmètre de protection, qui est unique, impose des servitudes à l'intérieur et à l'extérieur.

A l'intérieur du périmètre, les propriétaires des terrains concernés peuvent se voir interdire d'exécuter certains travaux sans autorisation ; ils peuvent également être contraints de supporter les travaux que le propriétaire de la source est en droit d'effectuer pour les besoins de son exploitation. A l'extérieur, en dehors du périmètre, et durant un délai de six mois avant la modification du périmètre, les propriétaires peuvent se voir imposer une interdiction d'accomplir certains travaux (article L 736 du code de la Santé). 


\section{LA MÉDIATION DU PROPRIÉTAIRE DU FONDS : UN PROCÉDÉ PLUS SOUPLE DE PROTECTION DE LA RESSOURCE EN EAU}

Deux orientations doivent être examinées en vue de compléter le dispositif de protection des captages qui passe par une meilleure maîtrise du foncier par le propriétaire et une action concertée sur l'activité agricole et les pratiques culturales.

\subsection{Une meilleure maîtrise du foncier}

Le propriétaire de terrains dont le tréfonds constitue une aquifere peut intervenir directement, en utilisant les techniques contractuelles classiques ; il peut également, en cas de location des terrains, orienter les dispositions du statut du fermage.

\subsubsection{Les techniques contractuelles classiques}

Afin d'éviter les inconvénients majeurs de la procédure d'expropriation (systématique dans le périmètre de protection immédiate, fréquente dans le périmètre de protection rapprochée (CE 13 décembre 1967)), le propriétaire des terrains dont le tréfonds renferme un aquifere propre à un captage, peut être incité à acquérir l'ensemble des terrains concernés par l'emprise, au besoin en faisant intervenir la SAFER (Société d'Aménagement Foncier et d'Etablissement Rural), dont l'une des missions réside dans l'aménagement du territoire.

Maîtrisant alors le foncier, le propriétaire des terrains pourra plus facilement en organiser la mise en valeur, en intervenant sur le mode de faire-valoir et sur l'activité ellemême.

Cette technique a été utilisée par le Groupe Perrier-Vittel pour protéger ses nappes d'eau minérale.

Parce qu'elle ne peut subir que des traitements très limités [8], la ressource en eau minérale doit être spécialement protégée.

Aussi, constatant que d'année en année, du fait des pratiques agricoles, une augmentation du taux de nitrate dans l'eau alimentant les deux nappes Vittel, le Groupe Nestlé, contrôlant la société des eaux de Vittel, a dès 1988, envisagé une démarche volontariste de protection du site hydrominéral.

En particulier, alors que la zone qui recueille les pluies alimentant les sources de Vittel s'étend sur 5000 hectares, dont 3500 de terres agricoles, le reste étant constitué par des massifs boisés, le Groupe Nestlé constatant que le danger venait des engrais azotés, s'est décidé à intervenir non seulement sur les pratiques culturales mais aussi et surtout sur le foncier. Ainsi a-t-il racheté 1620 hectares par l'intermédiaire de la SAFER qui les lui a rétrocédés dans le cadre des articles L 141-1 et R 142-2 du code rural, en vue d'une redistribution aux agriculteurs dans le cadre de convention de mise à disposition à titre gratuit, excluant de ce fait toute requalification au bail rural (à rapprocher des conventions de l'article L 4112 du code rural), et auxquelles a été annexé un cahier des charges dans les conditions de l'article R 142-2 imposant certaines pratiques culturales spécifiques définies en collaboration avec l'INRA (abandon de la culture du maïs, diminution du taux de chargement des prairies pâturées, valorisation des déjections animales, rotation des cultures...).

La mise en place de ces pratiques semble avoir été concluante, l'objectif de $10 \mathrm{mg} / \mathrm{litre}$ de nitrates ayant été atteint.
Enfin, l'intervention de la SAFER peut encore être envisagée dans le cadre de l'article L 412-6 qui permet à tout propriétaire de mettre à la disposition d'une SAFER, en vue de leur mise en valeur par des agriculteurs, des immeubles ruraux libres de location ; ces conventions échappent au statut du fermage mais leur durée est limitée à deux périodes de 6 ans.

\subsubsection{Les contraintes du faire-valoir indirect}

Lorsque la mise en valeur des parcelles à vocation agricole concernées par la protection de l'aquifere n'est pas faite directement par le propriétaire, mais par un exploitant dans le cadre du statut du fermage, la gestion de la protection de la ressource peut paraître plus difficile, dans la mesure où il est de l'essence du statut des baux ruraux que le preneur en place reste maître de son exploitation, des choix et orientations, et des techniques culturales mises en æuvre. Aussi peut-on craindre que les impératifs de productivité et de rentabilité l'emportent sur ceux tirés de la nécessité de protéger la ressource en eau contenue dans le tréfonds et aux alentours [9].

Le propriétaire n'est pourtant pas totalement dépourvu de moyens d'intervention.

D'une part, dans la mesure où des servitudes administratives existent dans la zone concernée, elles sont nécessairement opposables au preneur, de sorte que faute d'en respecter les contraintes, le propriétaire peut mettre en œuvre les sanctions habituelles, civiles et pénales. En particulier, il peut brandir les articles L 411-31 et L 411-53 du code rural, qui permettent la résiliation du bail en cas d'agissements de nature à compromettre la bonne exploitation du fonds ; il peut encore invoquer, notamment en fin de bail, l'article L 411-72 du même code qui permet au bailleur de demander des dommages-intérêts au preneur, en cas de dégradation du fonds, ce que peuvent constituer des techniques culturales excessivement polluantes.

D'autre part rien n'interdit d'imaginer un recours au dispositif de l'article L 411-32 du code rural qui permet au bailleur de résilier le bail en cas de changement de destination des parcelles, lorsque celles-ci se trouvent comprises dans une zone $\mathrm{U}$ du plan d'occupation des sols rendu public ou approuvé, ou lorsque le changement des destinations a été approuvé par le Préfet.

\section{- 3.2 Une action concertée sur l'activité agricole et les pratiques culturales}

Une autre orientation peut être envisagée - qui a déjà fait ses preuves - en matière de lutte contre les pollutions diffuses d'origine agricole, qui permet d'accompagner, voire d'anticiper la procédure pour garantir une protection du captage efficace : il s'agit d'intervenir directement sur l'activité agricole exercée sur les terrains situés dans la zone de protection. A cet égard, diverses mesures peuvent être envisagées, isolées ou conjuguées.

Des actions spécifiques en zone d'élevage intensif sont prévues dans des zones critiques d'alimentation des captages. A ce titre, une opération nationale de conseil en fertilisation (opération Ferti-mieux) a été conduite, qui engage les parties signataires d'une charte dans une démarche de labellisation d'opérations locales ou nationales destinées directement à conseiller les agriculteurs.

Mentionnons encore la mise en place par le décret du 27 août 1993 du code de bonne pratique agricole (CBPA) 
homologué par arrêté du 22 novembre 1993 dans le cadre de l'accord du 8 octobre 1993 proposé en application de la directive nitrate $\mathrm{n}^{\circ}$ 9/676 CEE du 19 décembre 1991, assorti d'un programme de formation visant à promouvoir sa mise en œuvre par les agriculteurs.

La réglementation des installations classées, essentiellement en ce qui concerne les installations d'élevage, participe également de cette orientation.

Dans le cadre du programme de maîtrise des pollutions agricoles, des aides peuvent également être apportées par les agences de l'eau, l'Etat et les collectivités locales, chaque éleveur devant signer un contrat individuel et s'engager à respecter la réglementation relative aux élevages.

Cette situation ne va pas, il est vrai, sans poser des difficultés, lorsque l'éleveur se trouve dans les liens d'un bail rural, la mise aux normes des bâtiments d'élevage, au regard, notamment des exigences en matière de lutte contre les pollutions, relevant en principe des "gros travaux", laissées par l'article L 415-10 à la charge du propriétaire bailleur [10]. Mais rien n'interdit, ici encore, aux parties de conclure un accord, aux termes duquel le preneur, exploitant, bénéficiant d'aides spécifiques [11], prendra à sa charge les travaux de mise aux normes.

La gestion de l'eau doit faire l'objet d'une attention accrue de la part des pouvoirs publics et se situer dans le cadre plus général d'une gestion durable des ressources en eau, y compris pour satisfaire les besoins de l'environnement.

C'est ce qui en France a été amorcé avec la loi sur l'eau du 3 janvier 1992 et son arsenal de dispositions réglementaires, qui permettent d'intervenir préventivement sur l'usage de la ressource car la qualité de l'eau et son approvisionnement n'ont pas de prix, encore qu'ils ont un coût ...!

Mais c'est également l'un des objectifs de la loi d'orientation agricole du 9 juillet 1999, qui vise "la préservation des ressources naturelles" et dont l'outil privilégié - le contrat territorial d'exploitation - doit "comporter un programme d'actions qui portent sur la contribution de l'activité de l'exploitation à la préservation des ressources naturelles".

\section{BILIOGRAPHIE}

[1] L'eau, gestion des ressources et protections de la qualité 1991 JO.

[3] R. LEMOAL et JP BEURIER. Statut juridique de la qualité de l'eau RDR 1996 p. 249 et s.

[4] A. HOULOU, L'eau : un droit , La vie judiciaire 4-5 juillet 1998

[5] B PEIGNOT - R.D.R. 1992 p.45 Les usages de l'eau à des fins non agricoles

[6] Directive CEE $n^{\circ} 80-778$ du 15 juillet 1980 révisée en 1997 et Décret du 3 janvier 1989 fixant les exigences de qualité et les normes en matière de potabilité

[7] J.L GAZZANIGA et X. LARROUY-CASTERA Eaux Jurisclasseur Rural - Fasc. Civ. 50

[8] D. FONTAINE, op. Cit. p. 4

[9] ef. notre communication au cours de la Première Conférence Droit de propriété et Environnement (1996) sur le thème : "Statut du fermage et de protection de l'environnement" Actes du colloque DALLOZ SIREY.

[10] Cass. Civ. 3ème 24 mars 1993 - D. 1993 IR p. 93 sur ces aides cf. spécialement Les Aides en agriculture par un groupe d'auteurs - Edition FRANCE AGRICOLE

[11] Sur ces aides cf. spécialement Les Aides en agriculture par un groupe d'auteurs - Edition FRANCE AGRICOLE. 